Article

\title{
Identification and Characterization of Seven Glutathione $S$-Transferase Genes from Citrus Red Mite, Panonychus citri (McGregor)
}

\section{Chong-Yu Liao ${ }^{\dagger}$, Kun Zhang ${ }^{\dagger}$, Jin-Zhi Niu, Tian-Bo Ding, Rui Zhong, Wen-Kai Xia, Wei Dou and Jin-Jun Wang *}

Key Laboratory of Entomology and Pest Control Engineering, College of Plant Protection, Southwest University, Chongqing 400715, China; E-Mails: leochongyu@gmail.com (C.-Y.L.); zhangkunbest@gmail.com (K.Z.); jinzhiniu@yahoo.com (J.-Z.N.); tianboding@gmail.com (T.-B.D.); zhongrui19890326@gmail.com (R.Z.); wenkaixia0409@gmail.com (W.-K.X.); anwdou@gmail.com (W.D.)

$\dagger$ These authors contributed equally to this work.

* Author to whom correspondence should be addressed; E-Mail: wangjinjun@swu.edu.cn; Tel.: +86-23-6825-0255; Fax: +86-23-6825-1269.

Received: 10 September 2013; in revised form: 21 November 2013 / Accepted: 22 November 2013 / Published: 13 December 2013

\begin{abstract}
The citrus red mite, Panonychus citri (McGregor), is a global citrus pest, and has developed severe resistance to several types of acaricides. However, the molecular mechanisms of resistance in this mite remain unknown. In this study, seven full-length cDNAs encoding glutathione $S$-transferases (GSTs) genes were identified and characterized in P. citri. The effects of pyridaben and fenpropathrin exposure on the expression of these genes were also investigated. Phylogenetic analysis revealed that the seven GSTs genes in P. citri cloned in this study belong to three different cytosolic classes, including four in mu, two in delta and one in zeta. Among these seven GSTs genes, the relative expression level of PcGSTm 1 was significantly higher in adult than in the other life stages (egg, larvae and nymph). Compared with the control, the mRNA levels of the seven GST genes did not change significantly following exposure to pyridaben at $\mathrm{LC}_{10}$. However, RT-qPCR results showed that, when exposed to $\mathrm{LC}_{10}$ of fenpropathrin, six GSTs gene (PcGSTm1, PcGSTm 3, PcGSTm4, PcGSTd1, PcGSTd2 and PcGSTz1) transcripts increased in a time-dependent manner. This is the first insight into the molecular characteristics of
\end{abstract}


GSTs gene cDNAs in P. citri. The elevated GSTs gene transcripts following exposure to fenpropathrin might be one of the mechanisms involved in detoxification of this acaricide.

Keywords: Panonychus citri; glutathione S-transferase; gene expression; pyridaben; fenpropathrin

\section{Introduction}

As one of the three major detoxification enzymes, glutathione $S$-transferases (GSTs; EC 2.5.1.18) are phase II metabolic enzymes capable of conjugating reduced glutathione to electrophilic centers of a wide range of exogenous and endogenous compounds [1]. GSTs can metabolize insecticides to more readily excreted water-soluble compounds by facilitating reductive dehydrochlorination or through conjugating with reduced glutathione [2]. It has been reported that GSTs play important roles in the development of pesticide resistance, disease pathogenesis, and cellular stress responses in mites and ticks [3-5]. For example, increases in GST transcripts have been investigated in scabies mites exposed to ivermectin [6]. Elevated levels of GSTs might play a protective role in Nilaparvata lugens, an insect species, following pyrethroid exposure [7]. GSTs can be induced not only by general inductive agent of this enzyme, but also by various insecticides [8,9]. In addition, GST gene expression levels have been shown to decrease in locusts when exposed to different concentrations of deltamethrin [10]. Thirty-five GST genes belonging to five different canonical GST classes (delta, epsilon, mu, omega and zeta) have been identified in the tick species Ixodes scapularis, which is a disease vector of veterinary and public health importance [11]. Annotation of the Tetranychus urticae genome has revealed the full extent of GSTs in Acari. The results of genome analysis showed that the delta and mu classes of GSTs exist as large gene clusters in two spotted spider mite GST superfamilies, but no epsilon class of GSTs has been identified in this species [12]. Moreover, 24 Unigene transcripts belonging to seven classes (delta, kappa, mu, omega, sigma, theta and zeta) have been identified from the transcriptome data of Panonychus citri [13]. Comparatively, in insects, GST superfamily genes have been clearly classified into at least six classes, including delta, epsilon, omega, sigma, theta and zeta [14].

The citrus red mite, Panonychus citri (McGregor) (Acari: Tetranychidae), is a major citrus pest distributed worldwide [15]. In the citrus orchards of southern China and Japan, the population has two peak time every year, one in early summer (June-July) and the other in autumn (October-November), while maintains low density during late summer and winter [16,17]. Moreover, owing to its unique biology and ecology, including a short life cycle, abundant progeny and arrhenotokous reproduction, the citrus red mite can survive frequent large-scale spraying of various acaricides/insecticides [18]. Since the first case of acaricide/insecticide resistance was reported in China in 1979, the citrus red mite has developed different degrees of resistance to various chemical classes of acaricides/insecticides (i.e., pyrethroids, abamectin, bifenazate, mitochondrial electron transport inhibitor acaricides and spirodiclofen) [19-21].

Enhanced enzymatic detoxification and target-site insensitivity are major causes of resistance in Acari [18]. Additionally, cytochrome P450 monooxygenases (P450s), carboxylesterase (CarE) and GSTs are the major detoxification enzymes involved in the metabolism of pesticides before they reach 
the target site [1]. However, there is limited research into acaricide/insecticide resistance mechanisms in P. citri relative to that in the model organism of Acari, $T$. urticae [18]. There have been some recent studies on detoxification enzymes that might be involved in development of resistance in $P$. citri, such as two cytochrome P450 genes, CYP4CF1 and CYP4CL2 [22]. Similarly, the results of acaricide challenge experiments showed that CarE genes, $P C E 1$ and $P C E 2$, may participate in the detoxification of avermectin and pyridaben [23]. By comparing the correlation coefficient of susceptibility of citrus red mite to pyridaben in nine field populations, along with their GST activities, it was suggested that GSTs could possibly be involved in detoxification of pyridaben in several field populations [24]. However, there is no available information about the relationship between GSTs and acaricides/insecticides at a molecular level in P. citri.

In our previous study, we identified 24 unique GST transcripts from annotation of the $P$. citri transcriptome [13]. To further characterize the molecular responses of GST genes to acaricides/insecticides in $P$. citri, in the current study we further obtained seven full-length GST cDNAs and carried out a detailed analysis of these genes. We then investigated potential differences in transcript expression levels that might be associated with acaricide metabolism and induction, and thus predicted functions of GST genes in P. citri by comparison with known GSTs in other arthropods.

\section{Results and Discussion}

\subsection{Identification and Classification of P. Citri GSTs}

In total, seven different GST transcripts were identified from the transcriptome database and were further aligned with the nucleotide sequences from genome datasets of T. urticae [25]. The complete, full-length cDNAs containing open reading frames were amplified by RACE-PCR, and the cDNA and deduced amino acid sequences were deposited in GenBank under the following accession numbers: JQ069034 (PcGSTm1), JQ069035 (PcGSTm2), JX846609 (PcGSTm3), JX846610 (PcGSTm4), JQ069033 (PcGSTd1), JQ069037 (PcGSTd2) and JQ069036 (PcGSTz1). Identity analysis and protein BLAST analysis of the predicted amino acid sequences of these GSTs suggested that the seven genes belonged to three different cytosolic classes, including four in mu (PcGSTm1, PcGSTm2, PcGSTm3 and PcGSTm4), two in delta (PcGSTd1 and PcGSTd2) and one in zeta (PcGSTz1) (Figure 1). The lengths of the deduced amino acid sequences of these seven $P$. citri GSTs varied from 217 to 224 a.a., and the predicted protein molecular weight ranged from 23.9 to $26.6 \mathrm{kDa}$, with theoretical isoelectric points of 5.18-7.65 (Table 1). The deduced amino acid sequence similarities were $11.4 \%-60.0 \%$ among all seven GSTs, $33.0 \%-60.0 \%$ among the four mu-class GSTs, and $53.2 \%$ between the two delta-class GSTs (Table 2). Among the different classes, the similarities of the deduced amino acid sequences were $11.4 \%-24.6 \%$ (Table 2). These results were consistent with the distribution of the seven putative amino acid sequences on the phylogenetic tree (Figure 1). However, based on transcriptome datasets, the remaining classes (kappa, omega, sigma and theta) of GST Unigene transcripts could not be amplified to full length in this study. This may be a result of low expression or the difficulty of designing primers according the restricted sequence lengths. However, the identified Unigene transcripts provide important information for a comprehensive understanding of the GSTs of $P$. citri. 
Figure 1. Phylogenetic relationship of 70 glutathione $S$-transferase (GST) proteins from six arthropod species, Tetranychus urticae (Tu, 31), Ixodes scapularis (Is, 16), Bombyx mori (Bm, 17), Drosophila melanogaster (Dm, 4), Anopheles gambiae (Ag, 1), Nasonia vitripennis $(\mathrm{Nv}, 3)$ and Tribolium castaneum (Tc, 1). Amino acid sequences were completely aligned using ClustalX, and a distance neighbor-joining tree was generated using MEGA 4.0. The seven $P$. citri GST genes are marked with filled brown circles.

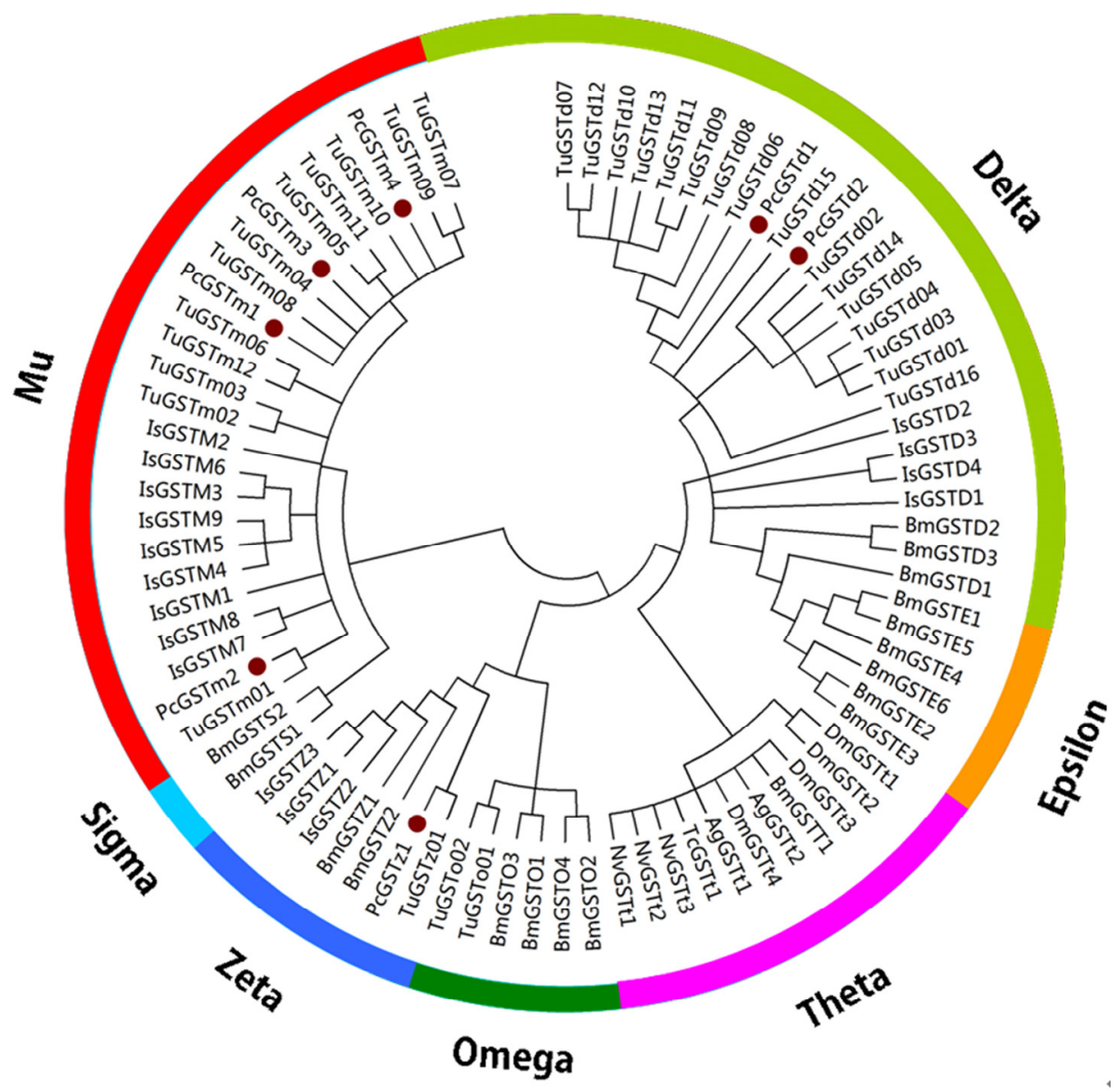

Table 1. Overview of molecular properties of seven P. citri GSTs.

\begin{tabular}{ccccccc}
\hline $\begin{array}{c}\text { Gene } \\
\text { name }\end{array}$ & $\begin{array}{c}\text { GenBank } \\
\text { accession number }\end{array}$ & $\begin{array}{c}\text { Length of } \\
\text { cDNA (bp) }\end{array}$ & $\begin{array}{c}\text { ORF } \\
\text { (bp) }\end{array}$ & $\begin{array}{c}\text { Number of deduced } \\
\text { amino acid residues }\end{array}$ & $\begin{array}{c}\text { Molecular } \\
\text { weight (kDa) }\end{array}$ & $\boldsymbol{p I}$ \\
\hline PcGSTm1 & JQ069034 & 1289 & 675 & 224 & 26.5 & 7.65 \\
$P c G S T m 2$ & JQ069035 & 1110 & 676 & 224 & 26.2 & 5.99 \\
$P c G S T m 3$ & JX846609 & 957 & 669 & 222 & 25.9 & 5.24 \\
$P c G S T m 4$ & JX846610 & 915 & 678 & 225 & 26.6 & 5.18 \\
$P c G S T d 1$ & JQ069033 & 905 & 654 & 217 & 23.9 & 6.52 \\
$P c G S T d 2$ & JQ069037 & 894 & 654 & 217 & 24.3 & 5.45 \\
$P c G S T z 1$ & JQ069036 & 822 & 657 & 218 & 24.7 & 6.91 \\
\hline
\end{tabular}


Table 2. Percentage identities of amino acid residues among the seven P. citri GST genes.

\begin{tabular}{lccccccc}
\hline & PcGSTm1 & PcGSTm2 & PcGSTm3 & PcGSTm4 & PcGSTd1 & PcGSTd2 & PcGSTz1 \\
\hline PcGSTm1 & - & 33.04 & 55.36 & 55.11 & 15.35 & 17.90 & 11.40 \\
PcGSTm2 & - & - & 37.89 & 35.24 & 19.57 & 18.10 & 17.30 \\
PcGSTm3 & - & - & - & 60.00 & 14.67 & 16.81 & 17.54 \\
PcGSTm4 & - & - & - & - & 13.10 & 13.97 & 14.89 \\
PcGSTd1 & - & - & - & - & - & 53.21 & 24.45 \\
PcGSTd2 & - & - & - & - & - & - & 24.56 \\
PcGSTz1 & - & - & - & - & - & - & - \\
\hline
\end{tabular}

\subsection{Comparison of GSTs from P. Citri and Other Species}

To date, all available GSTs have been classified into 13 different classes according to their sequence homology and molecular characteristics, and some GSTs are species-specific [26,27]. In Acari, total 35 GST genes belonging to seven classes (delta, epsilon, mu, omega, zeta, kappa and microsomal) have been found, based on analysis of the I. scapularis genome [11]. In addition, the genome dataset from $T$. urticae showed that all of the 31 cytosolic GST genes could be placed into four different classes: delta (16), mu (12), omega (2) and zeta (1) [12]. In this study, we only obtained seven full-length cDNAs encoding $P$. citri GST genes based on the transcriptome datasets, indicating that further investigation of remaining GSTs in P. citri is needed.

The delta class of GSTs is one of the two insect-specific GST classes, and members of this class can be found in the genomes of most insects, including Drosophila melanogaster [28], Anopheles gambiae [29], Aedes aegypti [30] and Bombyx mori [31] (Table 3). Although only two delta-class GSTs were obtained in the current study, the results are consistent with the previous prediction that this class of GSTs is likely to be widespread in Acari, as well as in Sarcoptes scabiei [32]. In addition, the popular understanding that delta-class GSTs are insect-specific may need to be reconsidered, with 16 delta-class GSTs identified from the genome of the two spotted mite, T. urticae. The existence of delta-class GSTs in $P$. citri could be related to the close taxonomical relationship between $P$. citri and T. urticae in Tetranychidae.

Mu-class GSTs have also been considered vertebrate-specific [33]. In mammals, mu-class GSTs are involved in catalyzing the reaction of glutathione with endo- and xenobiotics, and in a variety of cellular processes like detoxification of endogenous and exogenous compounds in the liver, brain, and testis [34,35] However, the abundance of mu-class GSTs in S. scabiei [6], I. scapularis [11], Varroa destructor and T. urticae has been reported [12,36]. Compared with other classes of this gene superfamily, the relative profusion of mu-class GSTs in both I. scapularis and T. urticae might suggest their more important role in the metabolism of xenobiotics across the whole lifecycle (Table 3). In this study, four complete cDNA sequences encoding mu-class GST genes were obtained. The phylogenetic tree clustered them with mu-class GSTs from T. urticae and I. scapularis (Figure 1). The deduced amino acid identities between the four P. citri mu-class GSTs and the mu-class GSTs in T. urticae were $54 \%-88 \%$, which was higher than in I. scapularis. These results were also consistent with their taxonomic status in Tetranychidae. 
Table 3. Comparison of the number of the GST genes from various insects and Acari.

\begin{tabular}{cccccccc}
\hline \multirow{2}{*}{ Cytosolic GSTs } & \multicolumn{4}{c}{ Insecta } & \multicolumn{4}{c}{ Acari } \\
\cline { 2 - 8 } & D. melanogaster & A. gambiae & T. castaneum & B. mori & T. urticae & I. scapularis & P. citri \\
\hline Delta & 11 & 12 & 3 & 4 & 16 & 7 & 2 \\
Epsilon & 14 & 8 & 19 & 8 & - & 5 & - \\
Mu & - & - & - & - & 12 & 14 & 4 \\
Omega & 5 & 1 & 4 & 4 & 2 & 3 & - \\
Sigma & 1 & 1 & 7 & 2 & - & - & - \\
Theta & 4 & 2 & 1 & 1 & - & - & - \\
Zeta & 2 & 1 & 1 & 2 & 1 & 3 & 1 \\
Unknown & - & 3 & - & 2 & - & - & - \\
Total & 37 & 28 & 35 & 23 & 31 & 32 & 7 \\
\hline
\end{tabular}

As in T. urticae, only one zeta-class GST was found in P. citri (Table 2), and their amino acid sequences shared $75 \%$ similarity. In the permethrin-resistant strain of B. mori, abundantly distributed zeta-class GSTs possibly contribute to the permethrin resistance of Lepidoptera [37]. However, little information about this class of GSTs in Acari is available.

\subsection{Developmental Stage-Specific Expression Patterns of GSTs in P. Citri}

Developmental stage-specific expression patterns of seven GST genes were examined in eggs, larvae, nymph, and adults using RT-qPCR. Among these seven genes, PcGSTm1 showed significantly higher expression (23.2-fold) in adult than in other developmental stages (Figure 2). That significant 10 fold greater expression of PcGSTm1 in adult could be considered as one key factor involved in the rapid development of acaricides resistance. Additionally, a higher relative expression level of GST at the larval stage compared with the adult stage has also been found in A. gambiae [38]. However, similar results have not been reported in mites and ticks. Interestingly, four mu-class GST genes (PcGSTm1, PcGSTm2, PcGSTm3 and PcGSTm4) had various expression patterns in four different developmental stages. However, significantly higher relative expression in adult than in larva was found for all mu-class GSTs, other than PcGSTm2. PcGSTm3 and PcGSTm4 showed lower expression in larva than in other stages. The lowest expression for PcGSTm2 (0.4-fold) was observed in nymph. The two delta-class genes (PcGSTd1 and PcGSTd2) showed similar expression profiles in the four developmental stages and had the lowest relative expression levels in adult and nymph, respectively (Figure 2). In addition, the lowest expression level of PcGSTz1 (0.37-fold) relative to eggs was observed in the larval stage (Figure 2). In summary, all of the genes were expressed at a higher level in adults than in larva and nymph, except PcGSTdland PcGSTd2. These results may be explained by differences in feeding rate and the various environmental challenges caused by mobility differences of $P$. citri during those stages. 
Figure 2. Developmental stage-specific expression patterns of the seven GST genes in Panonychus citri were evaluated using RT-qPCR. The following life stages were analyzed: egg, larvae, nymph and adult. The mRNA levels are expressed as mean fold transcription relative to egg. The letters above bars show significant differences among different developmental stages. The GAPDH gene was used as a reference. The differences among the four developmental stages were analyzed by one-way analysis of variance (ANOVA), followed by Duncan's multiple range tests in SPSS 16.0 (SPSS Inc, Chicago, IL, USA).
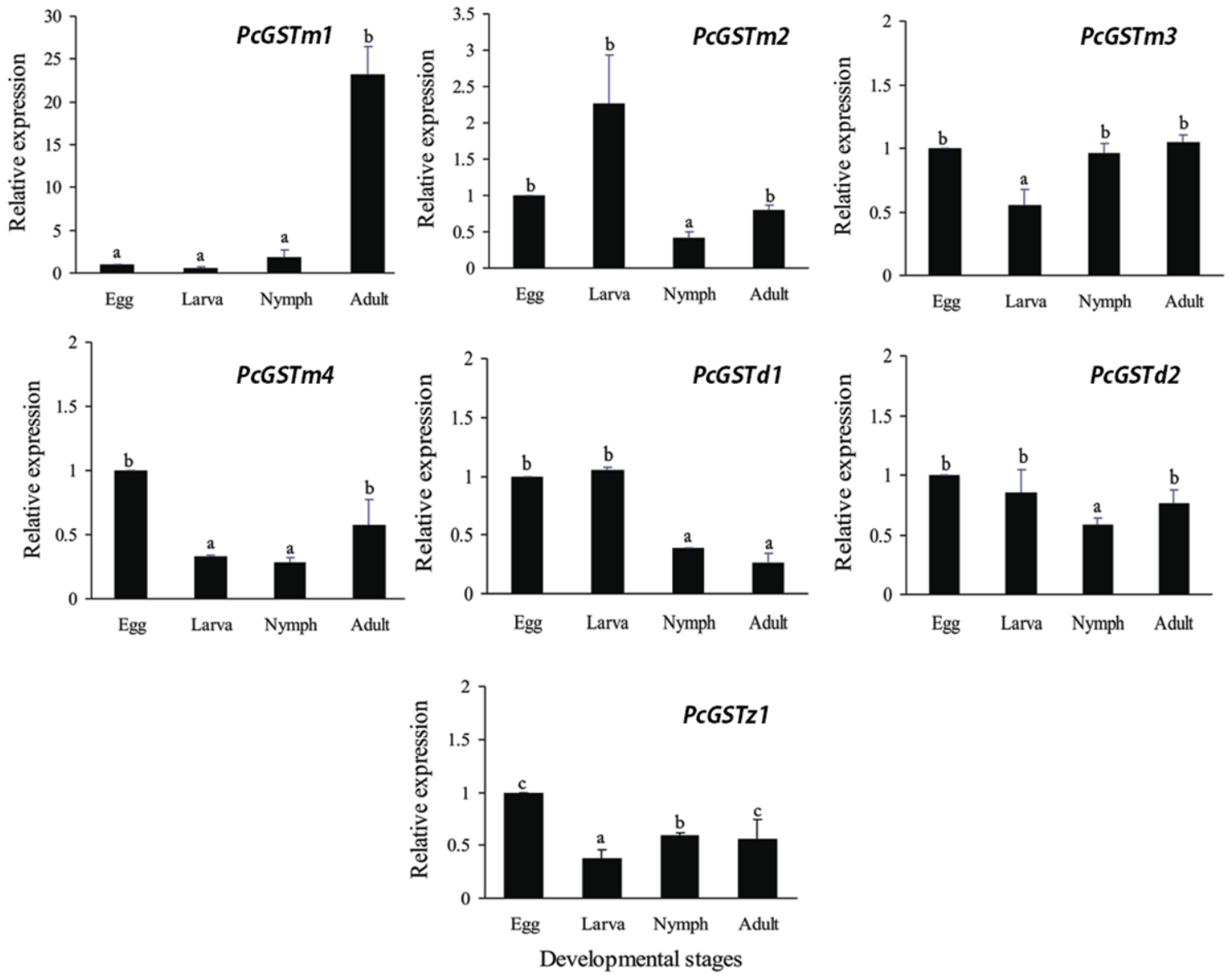

\subsection{Effect of Pyridaben and Fenpropathrin on GST Expression in P. citri}

Pyridaben, one of the mitochondrial electron transport inhibitor (METI) acaricides, is highly efficient against all life stages of spider mites, and is frequently used in many crops worldwide. METI resistance in $T$. urticae has been reported in several different regions and host plants [39]. The resistance against pyridaben has also been described in several strains of Panonychus ulmi [40]. A synergism study with diethylmaleate (DEM) and the measurement of P450 monooxygenase activity suggested the important factor in resistance to METIs is P450s, rather than glutathione $S$-transferases [41]. On the other hand, the susceptibility to pyridaben of $P$. citri has rapidly decreased over a short time in China [19]. Increased GST activity may be one of factors that contribute to the development of pyridaben resistance in P. citri [24]. To further reveal the relationship between GSTs and resistance to pyridaben in $P$. citri, we chose pyridaben as one of the two acaricides in this study to induce the expression of GST genes. Meanwhile, as a commonly-used acaricide in many orchards, fenpropathrin 
has a high resistance risk in P. citri [42]. The development of fenpropathrin resistance in $T$. urticae has been suggested to be related to the mutation and deletion of amino acid residues in the sodium channel $\alpha$-subunit gene (Tuvssc) [43]. However, in P. citri, elevated activities and/or expression of GST genes associated with resistance to fenpropathrin have not been reported to date.

In this study, induction results of the two acaricides indicated that none of the seven genes were significantly up-regulated following 12, 24, or $36 \mathrm{~h}$ of exposure to pyridaben (Figure 3A). The highest relative expression level was only 1.6 -fold after $36 \mathrm{~h}$ of exposure to pyridaben at $L C_{10}$. In field-collected pyridaben-resistant $P$. citri populations, synergism experiments with DEM, PBO and TPP suggested that GSTs play a major role in the development of pyridaben resistance [44]. In T. urticae, however, the inhibition study with DEM suggested that GSTs are not an important factor in resistance to pyridaben [41]. These differences may be explained by inter-specific differences [45]. Although the results in current study are consistent with a previous study in T. urticae we could not conclude for certain that GST is not an important factor in pyridaben tolerance, because the response of these seven GST genes to pyridaben exposure cannot completely represent the tendency of the whole supergene family. Therefore, remaining GSTs, especially those belonging to different classes, should be studied to determine whether they can be induced by pyridaben exposure in $P$. citri. Additionally, the concentration of pyridaben and exposure time might also impact the findings and therefore cannot be ruled out.

Interestingly, the relative expression levels of six GST genes (PcGSTm1, PcGSTm3, PcGSTm4, PcGSTd1, PcGSTd2 and PcGSTz1) in P. citri showed different time-dependent expression profiles when exposed to a sub-lethal concentration of fenpropathrin (Figure 3). Compared with the control, the relative expression levels of mu-class genes PcGSTm3 and PcGSTm4 were 4.1-fold and 9.6-fold higher soon after $12 \mathrm{~h}$ exposure to fenpropathrin. Another mu-class gene, PcGSTm1, also increased (2.4-fold) after $24 \mathrm{~h}$ of fenpropathrin exposure. No significantly increased relative expression level was found for PcGSTm2, suggesting its minor role in detoxification of fenpropathrin. Two delta-class GST genes, PcGSTd1 and PcGSTd2, were up-regulated 3.2-fold and 6.0-fold after $36 \mathrm{~h}$ of fenpropathrin treatment, respectively (Figure 3B). In addition, after $36 \mathrm{~h}$ of treatment with fenpropathrin, the relative expression levels of PcGSTm4 and PcGSTz1 also showed 7.3-fold and 3.0-fold increases, respectively.

The involvement of GSTs in pyrethroid resistance has been well studied. By binding to the molecule of pyrethroid insecticides in a sequestering mechanism, GSTs offer passive protection against pyrethroid [46]. Elevated GST levels may contribute to pyrethroid-induced lipid peroxidation to protect tissues from oxidative damage, and confer pyrethroid resistance in $N$. lugens [7]. However, little is known about GSTs that are involved in the resistance to pyrethroid acaricides. In the present study, three mu-class and two delta-class GST genes were induced following exposure to fenpropathrin (Figure 3B). This finding suggested that mu-class and delta-class GSTs in $P$. citri could be induced by fenpropathrin, and might play an important role in the development of resistance to fenpropathrin. Similar findings were obtained in permethrin-resistant S. scabiei [6], and are also consistent with several studies investigating the role of elevated GST activity in the development of resistance to pyrethroid insecticides in Aedes aegypti [47], Spodoptera littoralis [48] and Tribolium castaneum [49]. In addition that, zeta-class GST PcGSTz1 was also induced by fenpropathrin, similar to the mu- and delta-class GSTs. A similar result has been reported recently in B. mori, wherein GSTs were shown to catalyze the dechlorination of permethrin [36]. 
Figure 3. Quantification of relative expression levels of the seven GST genes in female adult Panonychus citri exposed to pyridaben (A) and fenpropathrin (B) at $L C_{10}$ for 12,24 and $36 \mathrm{~h}$ post-treatment. The mRNA level in the control and each treatment was normalized using GAPDH as a reference gene. The mean expression in each treatment was shown as a fold change compared with the mean expression in the control, which was defined as a basal value of 1 . The vertical bars indicate standard errors of the mean $(n=3)$. The asterisks on the bars indicate that means are significantly different among the control and treatments $(p<0.05)$.

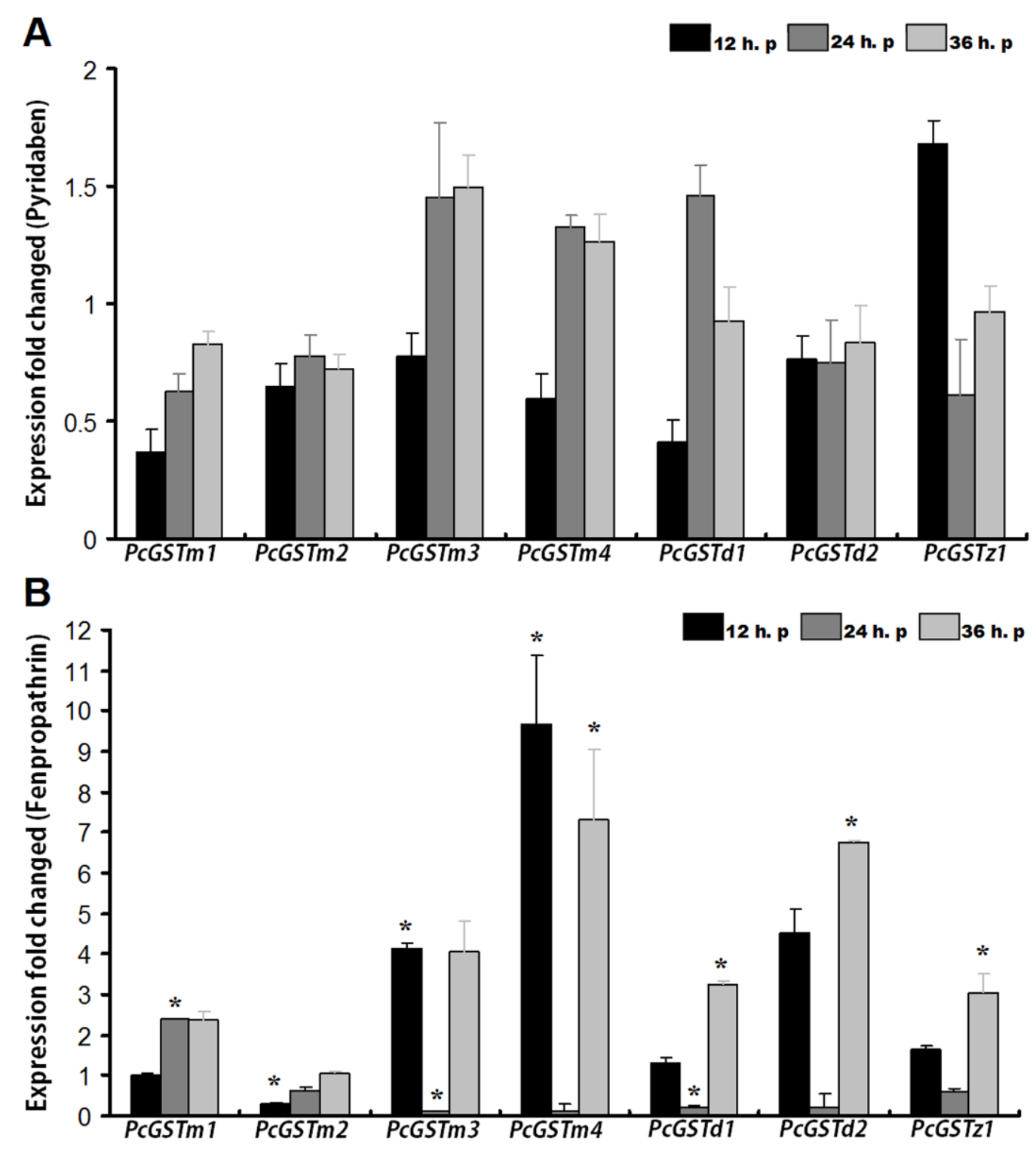

However, some of the seven GST genes showed decreased expression: PcGSTm2 (0.3-fold, $12 \mathrm{~h}$ treatment), PcGSTm3 (0.1-fold, $24 \mathrm{~h}$ treatment) and PcGSTd1 (0.2-fold, $24 \mathrm{~h}$ treatment). Among those, PcGSTm 2 expression recovered to generally the same level as the control after 24 and $36 \mathrm{~h}$ of fenpropathrin exposure. The non-induction response of PcGSTm2 to fenpropathrin exposure might suggest the discrepancies among different subclasses of GST genes. Dissimilar responses after various exposure times for these GST genes might be explained by a dynamic balance system to reasonably assign the energy among all the GST genes when challenged by xenobiotics. 
The increased expression of GST genes (PcGSTm1, PcGSTm3, PcGSTm4, PcGSTd1, PcGSTd2 and PcGSTz1) in P. citri might also result in an elevated tolerance to other pesticides and xenobiotics. Although the expression level of the remaining GST gene, PcGSTm2, was not significantly higher than the control when exposed to fenpropathrin, we speculate that this enzyme may be involved in resistance to other acaricides that were not tested rather than acaricides tested in the current study. Moreover, GSTs have not been shown to directly metabolize pyrethroid acaricides [2]. Therefore, further functional research of GSTs as detoxification enzymes or protector against resulting oxidative stress caused by xenobiotics is indispensable to elucidate the role of specific GSTs in fenpropathrin resistance development.

\section{Experimental Section}

\subsection{Mites}

The laboratory colony of $P$. citri was collected randomly from the Banco orchard at the Citrus Research Institute, Chinese Academy of Agricultural Sciences, Chongqing, China, in 2012. The collected mites were maintained at $25 \pm 1{ }^{\circ} \mathrm{C}$ and $60 \%$ relative humidity under a $14: 10 \mathrm{~h}$ light:dark regime. Mites at uniform developmental stages (including egg, larva, nymph and adult) were collected for RNA extraction, according to a previously-described collection method [22]. This population was relatively susceptible to both pyridaben and fenpropathrin based on results of laboratory bioassays together with a field population which has been challenged by acaricides for several years.

\subsection{Transcriptome Analysis and Identification of P. Citri GST cDNAs}

The GST genes from $P$. citri were identified by searching the Unigene transcriptome database [50] for keywords (GST, glutathione transferase and glutathione $S$-transferase) or by using the basic alignment search tool (BLAST) algorithm to search for other known arthropod GST genes. Unigene transcripts that were relatively short $(<300 \mathrm{bp}$ ) were manually removed and the remaining 24 Unigene transcripts were analyzed. To confirm the identity of the transcripts as GST genes, the putative GST cDNAs were subjected to BLASTP analysis to compare the predicted amino acid sequences against the non-redundant database at NCBI [50].

\subsection{RNA Isolation and First-Strand cDNA Synthesis}

Total RNA used for full-length cDNA amplification and analysis of the expression profiles of the GST genes was extracted using an RNeasy Plus Micro Kit (Qiagen GmbH, Hilden, Germany) according to the manufacturer's instructions. Genomic DNA was removed using a genomic DNA elimination column supplied with the kit. The total RNA from each sample was dissolved in $20 \mu \mathrm{L}$ of diethylpyrocarbonate-treated water and stored at $-80{ }^{\circ} \mathrm{C}$ for further analysis. RNA quantity was measured using the absorbance at $260 \mathrm{~nm}$ using a Nanovue UV-vis spectrophotometer (GE Healthcare, Fairfield, CT, USA), and the quality was assessed at an absorbance ratio of $O D_{260 / 280}$. The RNA integrity was further confirmed by estimating the $28 \mathrm{~S} / 18 \mathrm{~S}$ rRNA ratio by $2 \%$ agarose gel electrophoresis. The RNA for cDNA cloning and RT-qPCR was then reverse transcribed to synthesize first-strand cDNA using a PrimeScript 1st Strand cDNA Synthesis Kit (Takara Biotechnology Co., 
Dalian, China). The 5' and 3' cDNA regions were added using a SMARTer RACE cDNA Amplification Kit (Clontech, Mountain View, CA, USA) following the manufacturer's instructions.

\subsection{Cloning of Full-Length GST cDNA}

Based on the transcript Unigene sequences obtained from transcriptome searching, gene-specific primers were designed and synthesized (Table S1) for 5' and 3' rapid amplification of cDNA ends (RACE) analysis. Two universal primers, UPM (5'-CTAATACGGACTCACTATAGGGCAAG CAGTGGTATCAACGCAGAGT-3') and NUP (5'-AAGCAGTGGTATCAACGCAGAGT-3'), were also used. A pair of specific primers was designed to amplify the open reading frame for each gene after RACE analysis to confirm the full-length cDNA sequences (Table S2). The PCR conditions were determined empirically for amplification of each GST cDNA. They were $3 \mathrm{~min}$ at $95{ }^{\circ} \mathrm{C}$, followed by 34 cycles of $30 \mathrm{~s}$ at $95{ }^{\circ} \mathrm{C}, 30 \mathrm{~s}$ at $55-65{ }^{\circ} \mathrm{C}$ (depending on gene specific primers) and $60 \mathrm{~s}$ at $72{ }^{\circ} \mathrm{C}$, then $10 \mathrm{~min}$ at $72{ }^{\circ} \mathrm{C}$. The PCR products were purified from $1 \%$ agarose gel by Gel Extraction Mini Kit (Watson Biotechnologies Inc., Shanghai, China) and cloned into a pGEM-T Easy vector (Promega, Fitchburg, MA, USA). Inserts were further sequenced for confirmation (BGI, Beijing, China).

\subsection{Phylogenetic Analysis}

Deduced amino acids of obtained GSTs from P. citri, T. urticae, I. scapularis, and some of other model insects were aligned using ClustalX (v. 2. 0) [51]. The percentages of amino acid identity for each of the GSTs were determined using the MegAlign program (DNASTAR) [52]. The corresponding phylogenetic trees were determined by the neighbor-joining method, with 1000 bootstrap replicates implemented in MEGA 4.0 [53].

\subsection{Acaricide Exposures}

Fenpropathrin and pyridaben were purchased from Sigma-Aldrich (St. Louis., MO, USA) and used to induce GST gene expression according to the leaf disc immersion method [54]. The responses of GST genes to acaricides were investigated by exposing the female adult mites to these two acaricides at $\mathrm{LC}_{10}\left(10 \%\right.$ of lethal concentration) for 12,24 and $36 \mathrm{~h}$, respectively. The $L C_{10}\left(0.504 \mu \mathrm{g} \mathrm{mL}{ }^{-1}\right.$ for fenpropathrin and $0.250 \mu \mathrm{g} \mathrm{mL}^{-1}$ for pyridaben) for $P$. citri to the two acaricides was determined by leaf-dip bioassays prior to the acaricide treatments. Each leaf disc with 30 mites on the surface was soaked for $5 \mathrm{~s}$ in the acaricide solutions. For each treatment, more than 700 surviving mites were collected and three biological replicates were carried out. In addition, a corresponding number of mites were dipped in distilled water for $5 \mathrm{~s}$. All of the collected surviving mites were then rapidly frozen in liquid nitrogen and stored at $-80{ }^{\circ} \mathrm{C}$ for RNA extraction.

\subsection{Real-Time Quantitative PCR and Statistical Analysis}

All the primers for RT-qPCR were designed in Primer 3.0 [55] according to the full-length cDNAs obtained in this study (Table S3); based on a stability evaluation conducted in our previous study [56]. GAPDH was chosen to be the reference gene for RT-qPCR analysis of GST gene expression at four developmental stages and under acaricide exposures. The suitability of the primers for RT-qPCR 
was evaluated by a pre-experiment using general cDNA template from female adults. The amplification efficiency was determined by constructing a standard curve from a dilution series $(1,1 / 3$, $1 / 9,1 / 27$, and 1/81) of the cDNA samples. The RT-qPCR was performed using a Stratagene Mx3000P thermal cycler (Stratagene, La Jolla, CA, USA) with an initial denaturation at $95{ }^{\circ} \mathrm{C}$ for $120 \mathrm{~s}$, followed by 40 cycles of $95{ }^{\circ} \mathrm{C}$ for $15 \mathrm{~s}, 60{ }^{\circ} \mathrm{C}$ for $30 \mathrm{~s}$ and $72{ }^{\circ} \mathrm{C}$ for $30 \mathrm{~s}$. The relative expression level of the GST genes was calculated according to the $2^{-\Delta \Delta C t}$ method [57]. The differences among the four developmental stages were analyzed by one-way analysis of variance. The fold change of GST gene expression level was analyzed using SPSS (v.16.0, SPSS Inc, Chicago, IL, USA), and the significance was determined by independent-sample $t$-test with a $p$-value $<0.05$.

\section{Conclusions}

In this study, the full-length cDNA sequences of seven GST genes were identified from P. citri. Based on sequence analysis, these genes were placed into three different cytosolic classes, including four in mu, two in delta and one in zeta. In contrast to the dataset of the T. urticae genome, more GST genes in P. citri are expected to be identified in subsequent studies. Both increases and decreases in expression levels of the GST genes were observed when exposed to fenpropathrin at a sub-lethal concentration, whereas none of the seven GST was induced by pyridaben. These results may be caused by different mechanisms in the resistance development of $P$. citri to these two different types of acaricides/insecticides. Our work provides the first insight into the molecular characteristics and transcription profiles of $P$. citri GSTs when exposed to acaricides/insecticides.

\section{Acknowledgments}

This research was supported in part by the Special Fund for Agro-scientific Research in the Public Interest (201103020); the Program for Innovative Research Team in Universities (IRT0976); the National Natural Science Foundation (31171851); the earmarked fund for the Modern Agro-industry (Citrus) Technology Research System of China to Jin-Jun Wang; and the Fundamental Research Funds for the Central Universities (XDJK2013A017) of China to Wei Dou.

\section{Conflicts of Interest}

The authors declare no conflict of interest.

\section{References}

1. Li, X.C.; Schuler, M.A.; Berenbaum, M.R. Molecular mechanisms of metabolic resistance to synthetic and natural xenobiotics. Annu. Rev. Entomol. 2007, 52, 231-253.

2. Enayati, A.A.; Ranson, H.; Hemingway, J. Insect glutathione transferases and insecticide resistance. Insect Mol. Biol. 2005, 14, 3-8.

3. Dougall, A.; Holt, D.C.; Fischer, K.; Currie, B.J.; Kemp, D.J.; Walton, S.F. Identification and characterization of Sarcoptes scabiei and Dermatophagoides pteronyssinus glutathione $S$-transferases: Implication as a potential major allergen in crusted scabies. Am. J. Trop. Med. Hyg. 2005, 73, 977-984. 
4. Dreher-Lesnick, S.M.; Mulenga, A.; Mulenga, A.; Simser, J.A.; Azad, A.F. Differential expression of two glutathione $S$-transferases identified from the American dog tick, Dermacentor variabilis. Insect Mol. Biol. 2006, 15, 445-453.

5. Stumpf, N.; Nauen, R. Biochemical markers linked to abamectin resistance in Tetranychus urticae (Acari: Tetranychidae). Pestic. Biochem. Physiol. 2002, 72, 111-121.

6. Mounsey, K.E.; Pasay, C.J.; Arlian, L.G.; Morgan, M.S.; Holt, D.C.; Currie, B.J.; Walton, S.F.; McCarth, J.S. Increased transcription of glutathione $S$-transferases in acaricide exposed scabies mites. Parasites Vectors 2010, 3, doi:10.1186/1756-3305-3-43.

7. Vontas, J.G.; Small, G.J.; Hemingway, J. Glutathione $S$-transferases as antioxidant defence agents confer pyrethroid resistance in Nilaparvata lugens. Biochem. J. 2001, 357, 65-72.

8. Sivori, J.L.; Casabe, N.; Zerba, E.N.; Wood, E.J. Induction of glutathione $S$-transferase activity in Triatoma infestans. Memórias do Instituto Oswaldo Cruz 1997, 92, 797-802.

9. Yu, S.J.; Robinson, F.A.; Nation, J.L. Detoxication capacity in the honey bee, Apis Mellifera L. Pestic. Biochem. Physiol. 1984, 22, 360-368.

10. Qin, G.H.; Jia, M.; Liu, T.; Xuan, T.; Zhu, K.Y.; Guo, Y.; Ma1, E.; Zhang, J. Identification and characterisation of ten glutathione $S$-transferase genes from oriental migratory locust, Locusta migratoria manilensis (Meyen). Pest Manag. Sci. 2011, 67, 697-704.

11. Reddy, B.; Prasad, G.; Raghavendra, K. In silico analysis of glutathione $S$-transferase supergene family revealed hitherto unreported insect specific $\delta$-and $\varepsilon$-GSTs and mammalian specific $\mu$-GSTs in Ixodes scapularis (Acari: Ixodidae). Comput. Biol. Chem. 2011, 35, 114.

12. Grbić. M.; van Leeuwen, T.; Clark, R.M.; Rombauts, S.; Rouzé, P.; Grbić, V.; Osborne, E.J.; Dermauw, W.; Ngoc, P.C.; Ortego, F.; et al. The genome of Tetranychus urticae reveals herbivorous pest adaptations. Nature. 2011, 479, 487-492.

13. Niu, J.Z.; Dou, W.; Ding, T.B.; Shen, G.M.; Zhang, K.; Smagghe, G.; Wang, J.J. Transcriptome analysis of the citrus red mite, Panonychus citri, and its gene expression by exposure to insecticide/acaricide. Insect Mol. Biol. 2012, 21, 422-436.

14. Friedman, R. Genomic organization of the glutathione $S$-transferase family in insects. Mol. Phylogenet. Evol. 2011, 61, 924-932.

15. Gerson, U. Acarine pests of citrus: Overview and non chemical control. Syst. Appl. Acarol. 2003, $8,3-12$.

16. Shi, W.B.; Feng, M.G. Field efficacy of application of Beauveria bassiana formulation and low rate pyridaben for sustainable control of citrus red mite Panonychus citri (Acari: Tetranychidae) in orchards. Biol. Control 2006, 39, 210-217.

17. Furuhashi, K. Studies on population dynamics of citrus red mite, Panonychus citri McGregor in citrus orchards. Spec. Bull. Shizuoka Prefect. Citrus Exp. Stn. 1980, 4, 1-56.

18. Van Leeuwen, T.; Vontas, J.; Tsagkarakou, A.; Dermauw, W.; Tirry, L. Acaricide resistance mechanisms in the two-spotted spider mite Tetranychus urticae and other important Acari: A review. Insect Biochem. Mol. Biol. 2010, 40, 563-572.

19. Hu, J.F.; Wang, C.F.; Wang, J.; You, Y.; Chen, F. Monitoring of resistance to spirodiclofen and five other acaricides in Panonychus citri collected from Chinese citrus orchards. Pest Manag. Sci. 2010, 66, 1025-1030. 
20. Ran, C.; Chen, Y.; Wang, J.J. Susceptibility and carboxylesterase activity of five field populations of Panonychus Citri (Mcgregor) (Acari: Tetranychidae) to four acaricides. Int. J. Acarol. 2009, 35, 115-121.

21. Van Leeuwen, T.; Van Nieuwenhuyse, P.; Vanholme, B.; Dermauw, W.; Nauen, R.; Tirry, L. Parallel evolution of cytochrome $b$ mediated bifenazate resistance in the citrus red mite Panonychus citri. Insect Mol. Biol. 2010, 20, 135-140.

22. Ding, T.B.; Niu, J.Z.; Yang, L.H.; Zhang, K.; Dou, W.; Wang, J.J. Transcription profiling of two cytochrome $\mathrm{P} 450$ genes potentially involved in acaricide metabolism in citrus red mite Panonychus citri. Pestic. Biochem. Physiol. 2013, 106, 28-37.

23. Zhang, K.; Niu, J.Z.; Ding, T.B.; Dou, W.; Wang, J.J. Molecular characterization of two carboxylesterase genes of the citrus red mite, Panonychus citri (Acari: Tetranychidae). Arch. Insect. Biochem. Physiol. 2013, 82, 213-226.

24. Niu, J.Z.; Liu, G.Y.; Dou, W.; Wang, J.J. Susceptibility and activity of glutathione $S$-transferases in nine field populations of Panonychus citri (Acari: Tetranychidae) to pyridaben and azocyclotin. Fla. Entomol. 2011, 94, 321-329.

25. ORCAE. Online Rerource for Communnity Annotation of Eukaryots. Available online: http://bioinformatics.psb.ugent.be/orcae/overview/Tetur (accessed on 9 December 2013).

26. Dixon, D.P.; Lapthorn, A.; Edwards, R. Plant glutathione transferases. Genome. Biol. 2002, 3, doi:10.1186/gb-2002-3-3-reviews3004.

27. Sheehan, D.; Meade, G.; Foley, V.M.; Dowd, C.A. Structure, function and evolution of glutathione transferases: Implications for classification of non-mammalian members of an ancient enzyme superfamily. Biochem. J. 2001, 360, 1-16.

28. Sawicki, R.; Singh, S.P.; Mondal, A.K.; Benes, H.; Zimniak, P. Cloning, expression and biochemical characterization of one Epsilon-class (GST-3) and ten Delta-class (GST-1) glutathione $S$-transferases from Drosophila melanogaster, and identification of additional nine members of the Epsilon class. Biochem. J. 2003, 370, 661-669.

29. Ranson, H.; Claudianos, C.; Ortelli, F.; Abgrall, C.; Hemingway, J.; Sharakhova, M.V.; Unger, M.F.; Collins, F.H.; Feyereisen, R. Evolution of supergene families associated with insecticide resistance. Science 2002, 298, 179-181.

30. Lumjuan, N.; Stevenson, B.J.; Prapanthadara, L.A.; Somboon, P.; Brophy, P.M.; Loftus, B.J.; Severson, D.W.; Ranson, H. The Aedes aegypti glutathione transferase family. Insect Biochem. Mol. Biol. 2007, 37, 1026-1035.

31. Yu, Q.Y.; Lu, C.; Li, B.; Fang, S.; Zuo, W.; Dai, F.; Zhang, Z.; Xiang, Z. Identification, genomic organization and expression pattern of glutathione $S$-transferase in the silkworm, Bombyx mori. Insect Biochem. Mol. Biol. 2008, 38, 1158-1164.

32. Pettersson, E.U.; Ljunggren, E.L.; Morrison, D.A.; Mattsson, J.G. Functional analysis and localisation of a delta-class glutathione S-transferase from Sarcoptes scabiei. Int. J. Parasitol. 2005, 35, 39-48.

33. Chelvanayagam, G.; Parker, M.W.; Board, P.G. Fly fishing for GSTs: A unified nomenclature for mammalian and insect glutathione transferases. Chem. Biol. Interact. 2001, 133, 256-260. 
34. Campbell, E.; Takahashi, Y.; Abramovitz, M.; Peretz, M.; Listowsky, I. A distinct human testis and brain mu-class glutathione $S$-transferase. Molecular cloning and characterization of a form present even in individuals lacking hepatic type mu-isoenzymes. J. Biol. Chem. 1990, 265, 9188-9193.

35. Hussey, A.J.; Hayes, J.D. Human Mu-class glutathione $S$-transferases present in liver, skeletal-muscle and testicular tissue. Biochim. Biophys. Acta. 1993, 1203, 131-141.

36. Campbell, E.M.; Budge, G.E.; Bowman, A.S. Gene-knockdown in the honey bee mite Varroa destructor by a non-invasive approach: Studies on a glutathione $S$-transferase. Parasite Vectors 2010, 3, doi:10.1186/1756-3305-3-73.

37. Yamamoto, K.; Shigeoka, Y.; Aso, Y.; Banno, Y.; Kimura, M.; Nakashima, T. Molecular and biochemical characterization of a Zeta-class glutathione $S$-transferase of the silkmoth. Pestic. Biochem. Physiol. 2009, 94, 30-35.

38. Strode, C.; Steen, K.; Ortelli, F.; Ranson, H. Differential expression of the detoxification genes in the different life stages of the malaria vector Anopheles gambiae. Insect Mol. Biol. 2006, 15, 523-530.

39. Leeuwen, T.; Vontas, J.; Tsagkarakou, A.; Tirry, L. Mechanisms of Acaricide Resistance in the Two-Spotted Spider Mite Tetranychus Urticae. In Biorational Control of Arthropod Pests; Ishaaya, I., Horowitz, A. R., Eds.; Springer: Berlin, Germany, 2009; pp. 347-393.

40. Nauen, R.; Stumpf, N.; Elbert, A.; Zebitz, C.P.; Kraus, W. Acaricide toxicity and resistance in larvae of different strains of Tetranychus urticae and Panonychus ulmi (Acari: Tetranychidae). Pest Manag. Sci. 2001, 57, 253-261.

41. Van Pottelberge, S.; Van Leeuwen, T.; Nauen, R.; Tirry, L. Resistance mechanisms to mitochondrial electron transport inhibitors in a field-collected strain of Tetranychus urticae Koch (Acari: Tetranychidae). Bull. Entomol. Res. 2009, 99, 23-31.

42. Wang, J.; He, H. Resistance Selection and Risk Assessment of Fenpropathrin Against Panonychus citri (Acari: Tetranychidae). In Proceedings of APS-IPPC Joint Meeting, Honolulu, HI, USA, 6-10 August 2011; American Phytopathological Society: St. Paul, MN, USA, 2011.

43. Kwon, D.H.; Clark, J.M.; Lee, S.H. Cloning of a sodium channel gene and identification of mutations putatively associated with fenpropathrin resistance in Tetranychus urticae. Pestic. Biochem. Physiol. 2010, 97, 93-100.

44. Liu, Y.; Jiang, H. Resistance monitoring and synergism on four acaricides against Panonychus citri. J. Fruit. Sci. 2010, 27, 323-327.

45. Young, S.J.; Gunning, R.V.; Moores, G.D. The effect of piperonyl butoxide on pyrethroid-resistance-associated esterases in Helicoverpa armigera (Hübner) (Lepidoptera: Noctuidae). Pest Manag. Sci. 2005, 61, 397-401.

46. Kostaropoulos, I.; Papadopoulos, A.I.; Metaxakis, A.; Boukouvala, E.; Papadopoulou-Mourkidou, E. Glutathione $S$-transferase in the defence against pyrethroids in insects. Insect Biochem. Mol. Biol. 2001, 31, 313-319.

47. Grant, D.F.; Matsumura, F. Glutathione $S$-transferase 1 and 2 in susceptible and insecticide resistant Aedes aegypti. Pestic. Biochem. Physiol. 1989, 33, 132-143. 
48. Lagadic, L.; Cuany, A.; Bergé, J.B.; Echaubard, M. Purification and partial characterization of glutathione $S$-transferases from insecticide-resistant and lindane-induced susceptible Spodoptera littoralis (Boisd.) larvae. Insect Biochem. Mol. Biol. 1993, 23, 467-474.

49. Reidy, G.; Rose, H.; Visetson, S.; Murray, M. Increased glutathione $S$-transferase activity and glutathione content in an insecticide-resistant strain of Tribolium castaneum (Herbst). Pestic. Biochem. Physiol. 1990, 36, 269-276.

50. Wheeler, D.L.; Barrett, T. Database resources of the national center for biotechnology information. Nucleic Acids Res. 2007, 35, 5-12.

51. Larkin, M.; Blackshields, G.; Brown, N.P.; Chenna, R.; McGettigan, P.A.; McWilliam, H.; Valentin, F.; Wallace, I.M.; Wilm, A.; Lopez, R.; et al. Clustal W and Clustal X version 2.0. Bioinformatics 2007, 23, 2947-2948.

52. Clewley, J.P.; Arnold, C. MEGALIGN. In Sequence Data Analysis Guidebook; Springer: New York, NY, USA, 1997; pp. 119-129.

53. Tamura, K.; Dudley, J.; Nei, M.; Kumar, S. MEGA4: Molecular evolutionary genetics analysis (MEGA) software version 4.0. Mol. Biol. Evol. 2007, 24, 1596-1599.

54. Van Pottelberge, S.; van Leeuwen, T.; van Amermaet, K.; Tirry, L. Induction of cytochrome P450 monooxygenase activity in the two-spotted spider mite Tetranychus urticae and its influence on acaricide toxicity. Pestic. Biochem. Physiol. 2008, 91, 128-133.

55. Rozen, S.; Skaletsky, H. Primer3 on the WWW for General Users and for Biologist Programmers. In Bioinformatics Methods and Protocols; Stephen, M., Stephen, A.K., Eds.; Springer: Berlin, Germany, 1999; pp. 365-386.

56. Niu, J.Z.; Dou, W.; Ding, T.B.; Yang, L.H.; Shen, G.M.; Wang, J.J. Evaluation of suitable reference genes for quantitative RT-PCR during development and abiotic stress in Panonychus citri (McGregor) (Acari: Tetranychidae). Mol. Biol. Rep. 2012, 39, 5841-5849.

57. Livak, K.J.; Schmittgen, T.D. Analysis of relative gene expression data using real-time quantitative PCR and the $2^{-\Delta \Delta C t}$ Method. Methods 2001, 25, 402-408.

(C) 2013 by the authors; licensee MDPI, Basel, Switzerland. This article is an open access article distributed under the terms and conditions of the Creative Commons Attribution license (http://creativecommons.org/licenses/by/3.0/). 